\title{
Roll-to-Roll Cohesive, Coated, Flexible, High-Efficiency Polymer Light-Emitting Diodes Utilizing ITO-Free Polymer Anodes
}

\author{
Seongbeom Shin, Minyang Yang, L. Jay Guo, and Hongseok Youn*
}

This paper reports solution-processed, high-efficiency polymer light-emitting diodes fabricated by a new type of roll-to-roll coating method under ambient air conditions. A noble roll-to-roll cohesive coating system utilizes only natural gravity and the surface tension of the solution to flow out from the capillary to the surface of the substrate. Because this mechanism uses a minimally cohesive solution, the roll-to-roll cohesive coating can effectively realize an ultra-thin film thickness for the electron injection layer. In addition, the roll-to-roll cohesive coating enables the fabrication of a thicker polymer anode film more than $250 \mathrm{~nm}$ at one time by modification of the surface energy and without wasting the solution. It is observed that the standard sheet resistance deviation of the polymer anode is only $2.32 \Omega / \square$ over 50000 bending cycles. The standard sheet resistance deviation of the polymer anode in the different bending angles $\left(0\right.$ to $\left.180^{\circ}\right)$ is $0.313 \Omega / \square$, but the case of the ITO-PET is $104.93 \Omega / \square$. The average surface roughness of the polymer anode measured by atomic force microscopy is only $1.06 \mathrm{~nm}$. Because the surface of the polymer anode has a better quality, the leakage current of the polymer light-emitting diodes (PLEDs) using the polymer anode is much lower than that using the ITO-PET substrate. The luminous power efficiency of the two devices is $4.13 \mathrm{~lm} / W$ for the polymer anode and $3.21 \mathrm{~lm} / W$ for the ITO-PET. Consequently, the PLEDs made by using the polymer anode exhibited $28 \%$ enhanced performance because the polymer anode represents not only a higher transparency than the ITO-PET in the wavelength of $560 \mathrm{~nm}$ but also greatly reduced roughness. The optimized the maximum current efficiency and power efficiency of the device show around $6.1 \mathrm{~cd} / A$ and $5.1 \mathrm{~lm} / W$, respectively, which is comparable to the case of using the ITO-glass.

Prof. L. J. Guo, Dr. H. Youn

Department of Electrical Engineering and

Computer Science

The University of Michigan

Ann Arbor, Michigan, 48109, USA

E-mail: hgskyoun@umich.edu

S. Shin, Prof. M. Yang

Department of Mechanical Engineering

Korea Advanced Institute of Science and Technology

373-1 Guseong-dong, Yuseong-gu, Daejeon, 305-701, South Korea

DOI: $10.1002 /$ smll.201300382

\section{Introduction}

In recent years, flexible transparent conductive electrodes (TCEs) have been receiving much attention from many research groups due to its potential use in portable electronics, including electronic newspapers, flexible radio frequency identification devices, wearable solar cells, and flexible polymer light-emitting diodes (PLEDs). However, existing TCEs fabricated from materials such as indium tin oxide (ITO), aluminum zinc oxide (AZO), and indium zinc oxide (IZO) have critical problems. First, these metal oxides are so 
brittle that they have poor mechanical properties such as low flexibility. ${ }^{[1]}$ Even if those materials could be deposited on flexible substrates such as polyethylene terephthalate (PET), cracks occur during repeated bending motions. These cracks have a significant negative influence on the performance of flexible electronic devices. Second, indium, which is a generally used material in TCEs, is very vulnerable to poly(3,4-ethylenedioxythiophene) poly(styrenesulfonate) (PEDOT:PSS) acid. It is also expensive because it is a rare metal found only in some countries. ${ }^{[2]}$ In addition, the fabrication of metal oxide TCEs results in high production costs caused by the need for relatively complex processes such as a sputter system and a high-vacuum evaporator. Third, the indium forming the ITO diffuses into the upper layers of the TCEs, which leads to a decrease in device efficiency and life-time. ${ }^{[3]}$

Recently, single wall carbon nanotube (SWCNT) and silver nanowires were considered as the outstanding alternative for TCE materials. These conductive films showed the high transmittance and low sheet resistance at the same time. ${ }^{[4-9]}$ However, even though SWCNT and metal nanowires films are satisfied transmittance and sheet resistance, these films have a rough surface (roughness: 7 10 nm), which causes uneven film thickness of the upper layer and leakage current. Table S1 summarized material characteristics comparing ITO, conducting polymer, SWCNTs and silver nanowires. However, conducting polymers are one of the most favorable candidates for next-generation flexible TCEs due to their outstanding mechanical flexibility and high throughput using the Roll-to-Roll and other solution processes. Examples of conducting polymers that can be applied to flexible TCEs include poly(3,4-ethylenedi-

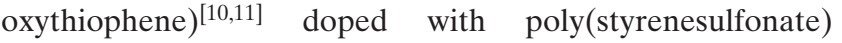
(PEDOT:PSS) and conductive polyaniline ${ }^{[12]}$ doped with camphorsulfonic acid (PANI:CSA). ${ }^{[13,14]}$ Although several flexible PLEDs have been demonstrated using these polymer materials, ${ }^{[15-17]}$ the performance of these devices, which are composed of polymer-based anodes, has been limited. Furthermore, several established PLEDs using polymer anodes are fabricated by multiple spin-casting processes to make a thicker film in order to attain higher conductivity. ${ }^{[18]}$ However, when the thicker film is spin coated, more solution wastage occurs. Thus, fabricating a polymer anode consumes significant amounts of material and requires a complicated process. A new process is definitely required that will enable us to make a thicker film in a single coating process.

The alternative process should enable us not only to fabricate a large-sized polymer anode on a flexible substrate but also to make a thicker film all at once without any solution wastage. Such a process would need to facilitate the fabrication of low-cost, high-quality devices while maintaining a uniform thickness across the whole device surface. Obtaining uniform thickness in the electrodes of devices such as PLEDs is crucial because thickness variations have a significant influence on current variations in the overall surface of PLEDs. These types of variations induce joule heating and breakage in the device, which causes degradation and shortening of the life of PLEDs. In addition, a thicker polymer anode has a lower sheet resistance. However, the transmittance of a thicker polymer anode is not enough to apply TCE of PLEDs because of low transmittance. In other words, high transmittances are conflict low sheet resistances, and the ideal value will be in only one point. The novel process should satisfy the above strict conditions.

Most previous studies have been limited in that they applied the solution process for fabricating only two layers, such as a hole injection layer (HIL) and an active layer. ${ }^{[19,20]}$ Also, previously used soluble electron injection materials such as $\mathrm{Cs}_{2} \mathrm{CO}_{3}{ }^{[21]}$ and $\mathrm{Ca}(\mathrm{acac})_{2}{ }^{[22]}$ have serious oxidation problems. In addition, various surfactant-like or water/ alcohol-soluble ionic polymers ${ }^{[23-26]}$ easily aggregate on the hydrophobic surface of a light-emitting polymer layer because most of these polymers are dissolved in polar solvents. ${ }^{[26]}$ In particular, when higher-molecular-weight polymers are used as light-emitting materials, the coating problems become more serious.

In this paper, $\mathrm{ZnO}$ nanoparticles (NPs) are used as the electron transport layer. They also supply a practical hydrophilic surface for an air-stable ionic solution containing ammonium cations as the electron injection layer. ${ }^{[27]}$

In the literature, the best current efficiency of the ITOfree device with PANI:PSA (drop-casted) as the transparent anode of, SY (spin-coated) as the light-emitting polymer, calcium as the electron injection layer (thermally evaporated) and aluminum as the cathode (thermally evaporated) was $5.6 \mathrm{~cd} / \mathrm{A}$, reported by Lee et al. ${ }^{[28]}$ Although all layers in the present device fabricated by the roll-to-roll cohesive coating under the ambient air condition, it exceeded $6.1 \mathrm{~cd} / \mathrm{A}$ even without the low work-function electron injection metal such as the calcium.

In terms of the roll-to-roll process, we should carefully select the solution process-able and air-stable the electron injection materials which do not contain alkali or alkalineearth metals at all. Among the various injection materials, the ammonium ion effectively form an interface dipole with an aluminium cathode within the $\mathrm{ZnO}$ nanoparticle/ammonium ion complex layer. Furthermore, the interface dipole can effectively lower the electron injection barrier. Therefore, the ammonium ion is useful not only to create interface dipole but also to be faricated by the roll-to-roll process.

We demonstrate a large-sized flexible PLEDs composed of polymer anodes using a solution process. The solution process, which is called roll-to-roll cohesive coating, consists of a main roll-to-roll system and an ink supply system. This coating system enables the fabrication of large-scale devices and the deposition of uniform layers at the same time. In addition, the roll-coating process has advantages in terms of controlling film thickness by regulating the roll speed, the spacer gap, the slit gap, the solution concentration, and the surface energy of the ink supply system and substrate. We investigate the roughness improvement and also report the optimal thickness of polymer anodes for maximum PLEDs efficiency in comparison with sheet resistance and transmittance of the polymer anode. Furthermore, the device performance utilizing a polymer anode fabricated by this roll-to-roll cohesive coating process, except for the Al cathode in an air atmosphere, shows outstanding efficiency compared to ITO-based flexible PLEDs and even ITO glass-based PLEDs. 


\section{Results and Discussion}

\subsection{Roll-to-Roll Coating Process for Large-Sized Flexible PLEDs}

In this work, our goal was to fabricate printable PLEDs. Among the various printing processes, the roll-to-roll coating system is considered to be the most favorable candidate for producing flexible, large-sized PLEDs. Because the use of a facile doctor blade added to the roll-to-roll system is a non-contact coating method with a simple configuration as compared to the slot-die system, it is a valuable tool for fabrication of large-sized thin films not only in the laboratory but also in industry. There is much research about PLEDs fabrication using a roll-coating system and a doctor blade. ${ }^{[20,21,29,30]}$ However, a serious problem with conventional blade coating has been the ink-supplying system, which could not deliver the ink on the substrate consistently. Therefore, the initial layer thickness was frequently thicker than the final layer thickness. To avoid this problem, Chen et al. employed hot blowing in a blade-only coating to make a uniform film. They reported that the uniformity of the film was around $10 \%$ in an area $50 \times 50 \mathrm{~mm} .^{[30]}$

In this study, however, to achieve better uniformity of the thinner organic layers such as the electron injection layer and to solve this fundamental ink-supplying problem, a commercial glass slide $(76 \times 25 \mathrm{~mm})$ was attached to the surface of a blade $(200 \mathrm{~mm} \times 28 \mathrm{~mm})$ composed of thermally annealed stainless steel, as shown in Figure 1(a),(b). The solution contained in the slit capillary began to flow and initially created a meniscus, followed by a homogeneous laminar flow. This fine laminar flow is the key to uniformity and better film quality. The control variables of the film thickness are the blade gap, the slit gap, the blade speed, the ink concentration, and the surface energy of the substrate and the slit.

It has already been noted that most previous studies have been limited in that they have applied the solution process for fabricating only two layers, such as a hole injection layer (HIL) and an active layer. ${ }^{[20,21]}$ Because the PEDOT:PSS and light-emitting polymer layers are relatively much thicker than the electron injection or the electron transport layers, they are much easier to fabricate by using typical solution processes. Thus, to fabricate these thinner layers successfully requires a much smaller feeding capacity of the solution. In the PLEDs described in this paper, the total thickness of the $\mathrm{ZnO} \mathrm{NP/}$ ionic complex layers, serving as the electron transport/electron injection layers, respectively, is approximately $15-30 \mathrm{~nm}$.

A conventional slot-die system can be a good candidate for fabricating organic electronic devices. ${ }^{[31]}$ However, it is very difficult to realize the thinner electron transport/injection layer utilizing a conventional slot-die pumping system. Therefore, to reduce and homogenize the flow-rate of the coating solution effectively through the ink delivery system, some groups employed an additional ink delivery system instead of the ink delivery system used in a conventional slotdie coater using a static pump. ${ }^{[32]}$

In this paper, the facile roll-to-roll coating system does not require an external pumping system. The roll-to-roll
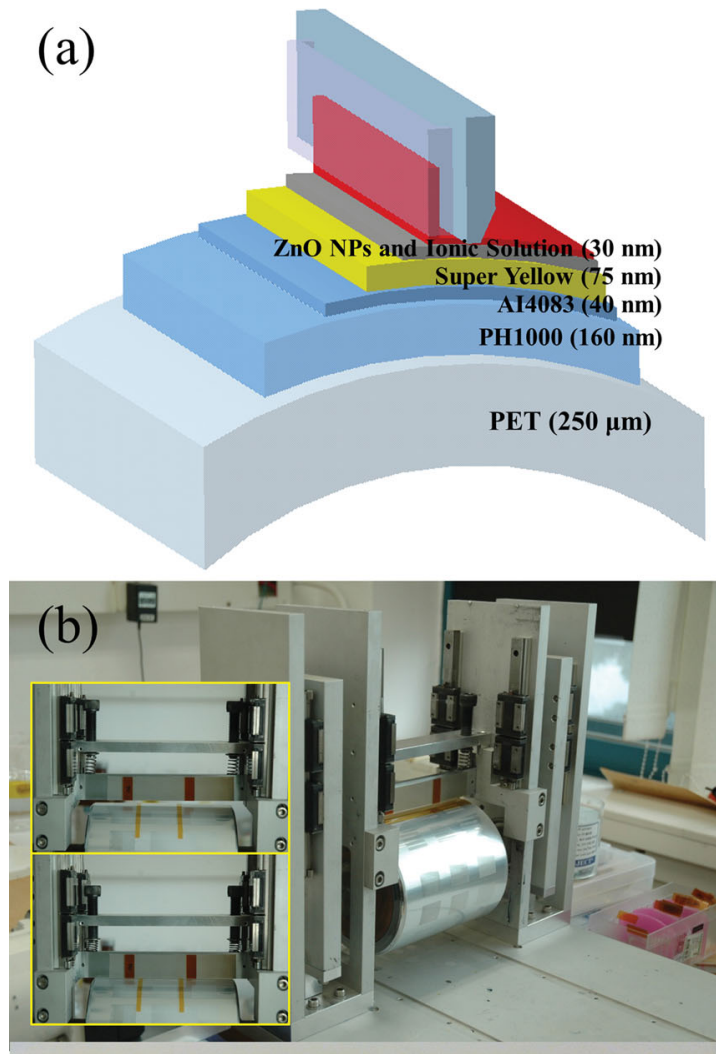

(c)

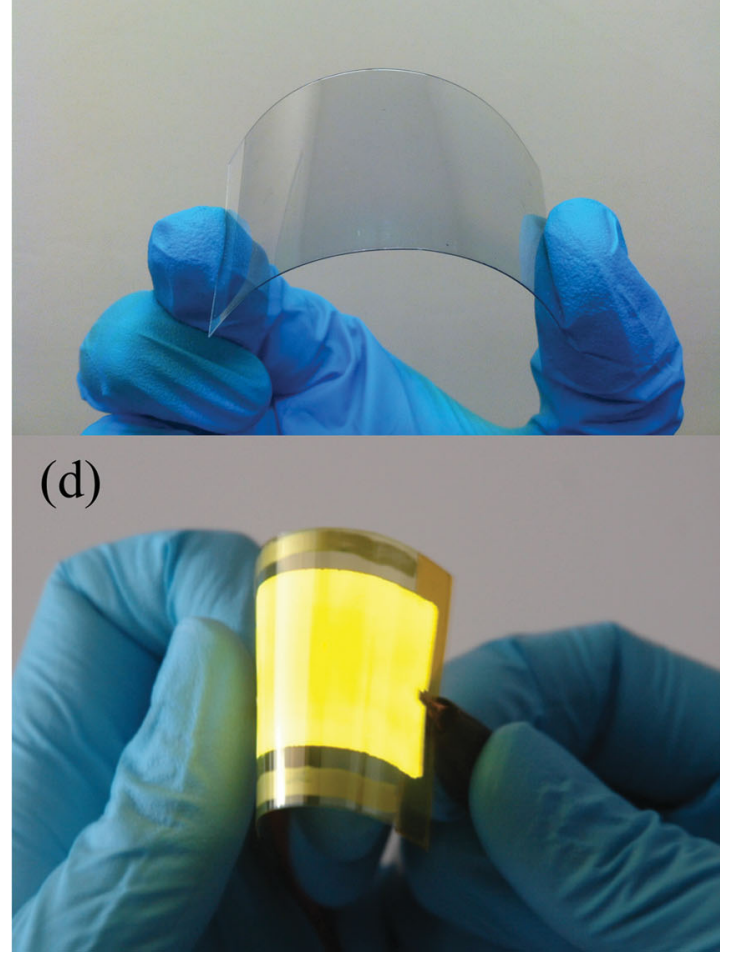

Figure 1. (a) Illustration of the device structure and the configuration of the roll-to-roll cohesive coating process. (b) The image of the rollto-roll cohesive coating system and composed of roller, doctor blade, and slit nozzle system. (c) The roll-to-roll coated polymer anode film. (d) The yellow light-emitting flexible PLEDs on the PET substrate, size $30 \mathrm{~mm} \times 40 \mathrm{~mm}$. 
cohesive coating system described in this work utilizes only natural gravity and the surface tension of the solution to flow out from the slit capillary to the surface of the substrate. At first, the solution creates the coating bead due to the gravity of the solution and the surface tension between the blade and the substrate. When the nozzle moves in the coating direction, the solution automatically starts to flow out from the slit gap space to the substrate by action of the cohesive force of the solution itself, as shown in Figure 2(b),(c). However, if the moving speed is too fast to maintain the coating bead kept by the cohesive force of the solution, the wet-coating film will be broken. However, the roll-to-roll cohesive coating can effectively reduce the flow rate and the wet-film thickness because this mechanism uses the minimal cohesive energy of the solution. The amount of the solution for each layer to be coated is only $25 \mu \mathrm{L}$ in an area $50 \mathrm{~mm} \times 50 \mathrm{~mm}$. The whole volume of the slit capillary space is $550 \mu \mathrm{L}$.

A brief description of the fabrication processes is as follows. Every layer was fabricated by the roll-to-roll cohesive coating process at a room temperature of $20{ }^{\circ} \mathrm{C}$ under ambient air conditions. The spacer, which is a polyimide film about $70 \mu \mathrm{m}$ thick, was attached to both edges of the PET at every step. Before the coating processes, in order to modify the surface energy and make a uniform surface for homogenous flow in the slit capillary space, the surfaces of the slide glasses were treated by using oxygen plasma exposure or self-assembled monolayer (SAM) deposition. A typical SAM material is $n$-octadecyltrichlorosilane (OTS). The large surface energy of the slit decreases the flow rate effectively, and the small surface energy utilizing SAMs increases the flow rate of the solutions. Because the solvent for PEDOT:PSS is water, consisting of hydrogen and oxygen, it has high polarity. Therefore, the water-based PEDOT:PSS solution also has high polarity and a very large adhesive force between the hydrophilic surface and its molecules. To make a thicker polymer anode film for increased conductivity with a one-time coating, we simply added an additional SAM. On the other hand, in case of the hydrophobic surface using the SAM, the cohesive forces $\left(F_{\mathrm{c}}\right)$ among the molecules are relatively higher than the adhesive force $\left(F_{\mathrm{a}}\right)$ between the surface and the molecule [Figure 2(a)]. Therefore, the flow rate will be effectively increased. We used this phenomenon to control the flow rate for various solutions ejected by the slit outlet. Moreover, to create ultrathin layers such as the $\mathrm{ZnO}$ nanoparticle layer and the ionic layer, the glass slide and blade surface were exposed to oxygen plasma [see the red surface of Figure 2(b)]. On the other hand, for increasing the thickness of the coated film, the SAM was deposited on three components of the slit nozzle system [see the blue surface of Figure 2(c)]. Furthermore, surface energy modification is helpful not only for controlling the adhesive and cohesive forces but also for controlling the surface energy in order to obtain a homogeneous coating and uniform ink injection.

This method is inexpensive, and it is easy to change and clean the nozzle for the different solutions. Because our blade-slot coating system employs a transparent glass slide for the slot capillary, the slide is quite useful for observing the fluid flow into the slot nozzle.
Molecule (partially $\delta^{-}$or $\delta^{-}$)

Cohesive force between liquid and liquid $\left(\boldsymbol{F}_{\mathrm{c}}\right)$

Adhesive force between liquid and surface $\left(\boldsymbol{F}_{\mathrm{a}}\right)$

Surface modification

(Self-Assembled Monolayers, $\mathrm{O}_{2}$ Plasma, Teflon film)

- Low-surface-energy region (Downward concavity)

- High-surface-energy region (Up ward concavity)

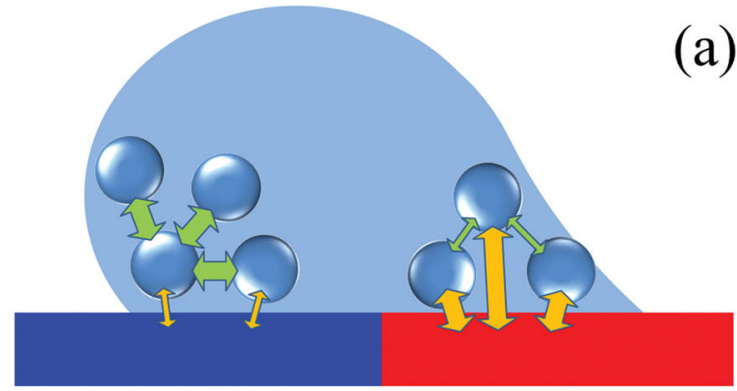

Downward concavity $\left(\boldsymbol{F}_{\mathrm{c}}>\boldsymbol{F}_{\mathrm{a}}\right)$

Upward concavity $\left(\boldsymbol{F}_{\mathbf{c}}<\boldsymbol{F}_{\mathrm{a}}\right)$
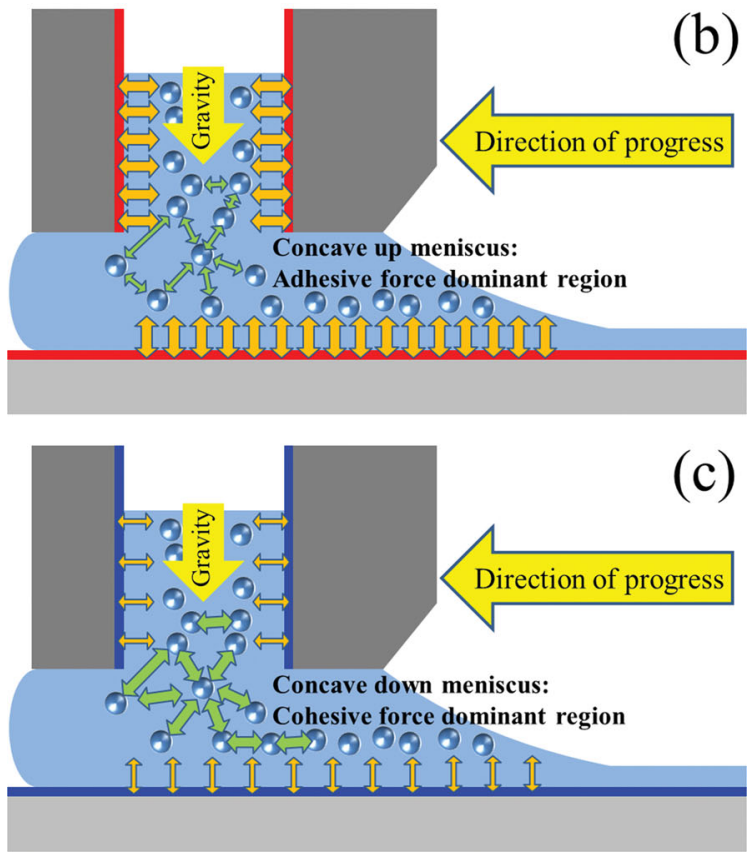

Figure 2. Illustrations of the cohesive coating mechanism and coating flows details. (a) Adhesive $\left(\boldsymbol{F}_{\mathbf{a}}\right)$ and cohesive $\left(\boldsymbol{F}_{\mathbf{c}}\right)$ force diagram between hydrophobic and hydrophilic surfaces. Contact angles depend on the relative forces between solvent molecules and surfaces. In the case of downward concavity, a cohesive force is higher than an adhesive force, whereas upward concavity represents that an adhesive force is higher than a cohesive force. (b) The case of adhesive force dominant condition. Adhesive forces are enhanced by surface energy modification such as $\mathrm{O}_{2}$ plasma treatment. Therefore, the flow rate is reduced by adhesive forces. (c) The case of cohesive force dominant condition. Adhesive forces are relatively reduced by self-assembled monolayers (SAMs). Thus, the flow rate is increased.

Based on previous studies, we demonstrate a completely solution-processed PLEDs, from the polymer anode to the electron injection layer, using a roll-coating system composed of a roll coater, doctor blade, and slot. Furthermore, 

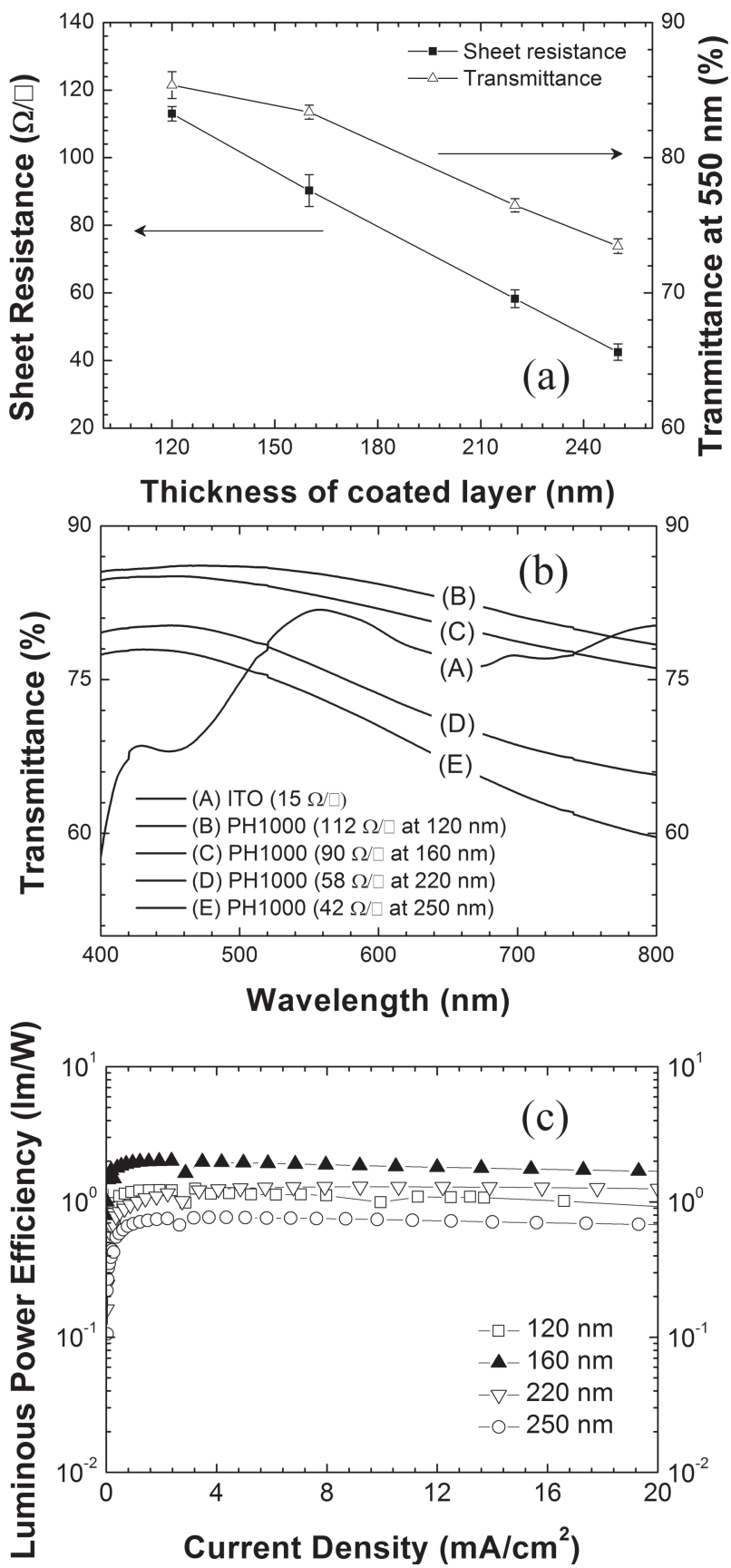

Figure 3. Polymer anode thickness optimization for high-performance PLEDs. (a) Variation of the sheet resistances and the transmittances following thicknesses of polymer anode thin films, coated by roll-toroll cohesive coating method. (b) Transmittance comparison between ITO anode film and polymer-anode films in visible wavelength. (c) The luminous power efficiency variation according to the thicknesses of polymer-anode thin films.

the roll-coating system facilitated the achievement of better surface roughness and uniformity. Figure 1(b) shows the configuration of the roll-to-roll cohesive coating system. The glass slide was attached to the doctor blade, which was subsequently attached to the roll system. The slit capillary contains the solutions and then discharges the solutions through the nozzle. The system does not employ an external solution feed system.

\subsection{Characteristics of Roll-to-Roll Coated Polymer Anode}

The electrical conductivity of TCE depends on its thickness. Generally, the conductivity of TCE declines significantly when its thickness decreases. In addition, the thickness is related to the transmittance of TCE. These two factors, the conductivity and the transmittance, have a conflicting relationship. Therefore, the optimal conditions of the two factors have to be decided in order to fabricate high-efficiency PLEDs. In addition, TCE, as a bottom anode in the lightemitting diodes, has to achieve high transmittance and low sheet resistance at the same time. In this work, we found the optimum thickness of a polymer andoe to be approximately $160 \mathrm{~nm}$ using the roll-coating process.

Figure 3(a) shows that the sheet resistances and transmittances are directly correlated to the thickness of the polymer anode. This means that the maximum efficiency is located at the inflection point, as represented in Table 1. The thickness of $160 \mathrm{~nm}$ satisfies the minimized absorption of emission light and provides enough conductivity for the anodes at the same time. Figure 3(b) in particular indicates that the optimal polymer anode $(\mathrm{C})$ is superior to an ITO anode in transparency near ultraviolet and visible ranges. The low transparency of these ranges of ITO has been a crucial problem for commercialization.

The sheet resistance variance during the bending motions of TCEs is the most important factor for flexible electronics. The sheet resistance of the ITO film was severely affected with respect to various bending angles. The sheet resistance of the ITO-PET increased significantly at angles of approximately $180^{\circ}$. This implies that the ITO film was cracked by the bending stress. However, in the case of the polymer anode, there were no serious changes. The anode exhibited robustness to the bending stress, as shown in Table 2.

In addition, we observed that the sheet resistance was maintained during 50000 continuous bending cycles. The bending curvature was $0.2 \mathrm{~mm}^{-1}$, and the experiment setup is shown in Figure 4. During the bending cycles, the sheet resistances of the polymer anode were approximately from 64 to $70 \Omega / \square$, which were acquired by a four-point probe measurement system. The result of the sheet resistance variance thus demonstrates the bending stability of the polymer anode.

Table 1. The characteristic of polymer anode film with respect to the thickness change. The sheet resistances are decreased along with the thickness increase of the conducting polymer film. The transmittances are also decreased. In other way, the maximum efficiency has only one optimal condition in terms of polymer anode thickness: about $160 \mathrm{~nm}$. Transmittance values include the PET substrate and are compared with air.

\begin{tabular}{lcccc}
\hline & $120 \mathrm{~nm}$ & $160 \mathrm{~nm}$ & $220 \mathrm{~nm}$ & $250 \mathrm{~nm}$ \\
\hline $\begin{array}{l}\text { Sheet resistance } \\
{[\Omega / \square]}\end{array}$ & 112.5 & 90.4 & 58.1 & 42.5 \\
$\begin{array}{l}\text { Transmittance at } \\
550 \mathrm{~nm}[\%]\end{array}$ & 85.4 & 83.4 & 76.6 & 73.6 \\
$\begin{array}{l}\text { Max. Efficiency } \\
{[\mathrm{Im} / \mathrm{W}]}\end{array}$ & 1.25 & 2.01 & 1.30 & 0.77 \\
\hline
\end{tabular}


Table 2. The sheet resistance change of conducting polymer anode and ITO-PET following the change in bending angle. The sheet resistance of ITO is significantly increased when the bending angle is increased. The conducting polymer shows almost the same sheet resistance in bending motion.

\begin{tabular}{lccccccc}
\hline & $0^{\circ}$ & $30^{\circ}$ & $60^{\circ}$ & $90^{\circ}$ & $120^{\circ}$ & $150^{\circ}$ & $180^{\circ}$ \\
\hline ITO PET $[\Omega / \square]$ & 13.9 & 17.6 & 26.1 & 40.3 & 52.6 & 68.2 & 309.3 \\
Polymer & 68.8 & 69.0 & 69.0 & 69.1 & 69.2 & 69.5 & 69.7 \\
anode $[\Omega / \square]$ & & & & & & & \\
\hline
\end{tabular}

Another advantage of the roll-coated polymer anode was the improvement on the surface. As a thin-film PLEDs, the roughness of the substrate and the anode had a significant influence on device efficiency and life-time because a nonuniform surface could generate the leakage charge between the anode and cathode. ${ }^{[33,34]}$ The average surface roughness of the ITO film on the PET substrate was $3.75 \mathrm{~nm}$, whereas the average surface roughness of the polymer anode film was only $1.06 \mathrm{~nm}$. The maximum roughness of the films was $63.0 \mathrm{~nm}$ (TIO-PET) and $9.97 \mathrm{~nm}$ (polymer anode), respectively. The roughness of each surface was determined by AFM. The surface wrinkle seen in the ITO film was created during the ITO sputtering process, as shown in Figure 5(a). In particular, it has been reported that the plasma wrinkles the surface of polymer substrates such as PET. ${ }^{[35]}$ In the case of ITO, its surface roughness is determined when it is produced and its value remains constant during the process. However, our device structure and processing system can improve surface roughness because PH1000 and AI4083 (hole injection material) are composed of the same solvent and compound. Figure 5(b) shows the typical roughness of the polymer anode without AI4083. Furthermore, HOMO (highest occupied molecular orbital) levels of PH1000 and AI4083 closely matches than the case of ITO and AI4083.

In terms of the life-time stability, the polymer anode is vulnerable to moisture and oxygen in an air atmosphere, as shown in Figure 6. The sheet resistance was degraded with respect to the exposure time. The sheet resistance of the

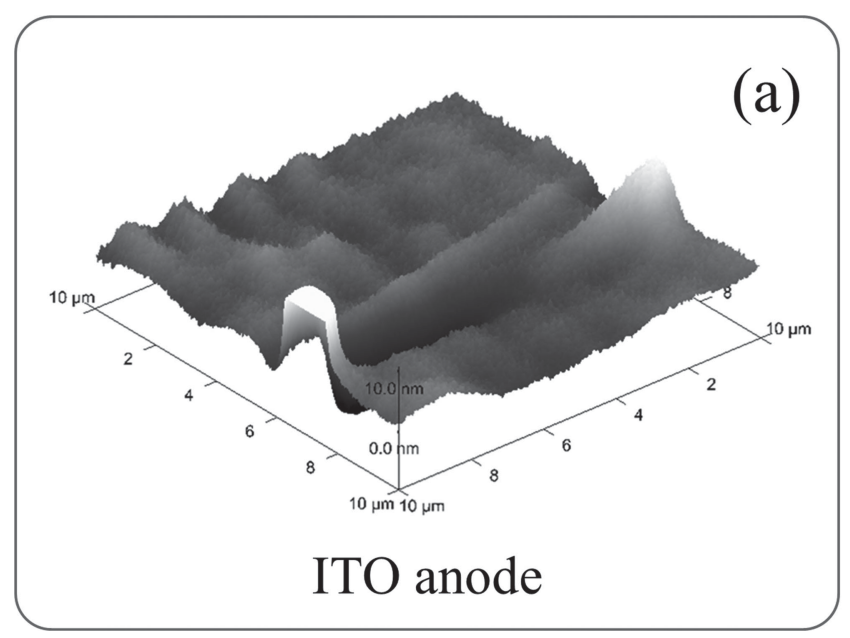

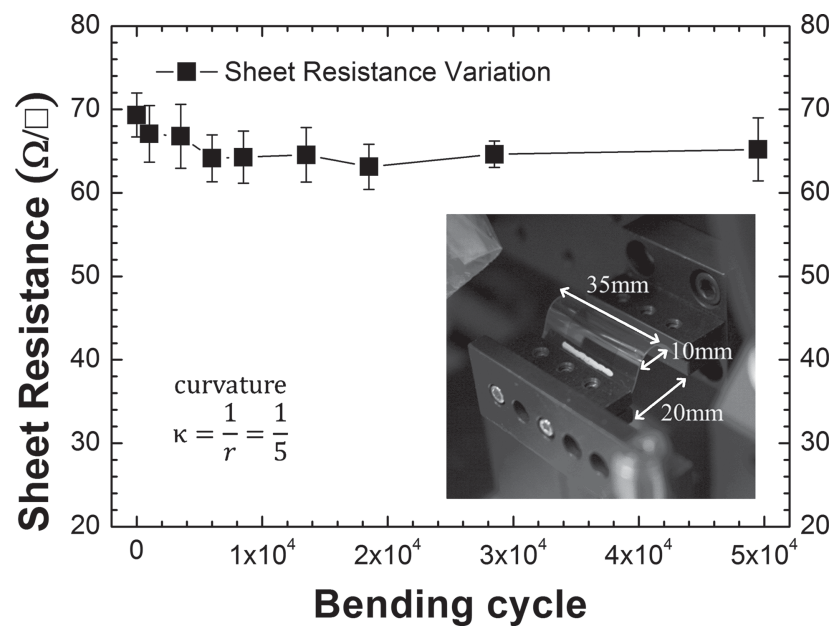

Figure 4. The sheet resistance variation of the polymer anode during the bending cycle. And the experiment configuration and dimensions, represented by curvature (inset). The repetitive motion began to bend at flat neutral condition and the maximum curvature was $0.2 \mathrm{~mm}^{-1}$.

polymer anode increased $50 \%$ after $500 \mathrm{~h}$ of air exposure time. On the other hand, the sheet resistance of the polymer anode kept in the nitrogen-filled glove box maintained its resistance for more than $515 \mathrm{~h}$. Therefore, to increase the stability against the moisture and oxygen, the polymer anode should have additional encapsulation layers. In addition, the life-time of PLEDs encapsulated by glass was around $400 \mathrm{~h}$ as shown in Figure S3.

\subsection{Results of Device Performance}

Recently, there are many kinds of high efficient PLEDs, ${ }^{[36]}$ which are commonly fabricated by the vacuum evaporation process. Also, small molecular OLEDs and phosphorescent OLEDs could have already achieved extremely high efficiency. ${ }^{[37]}$ On the other hand, this paper focuses on

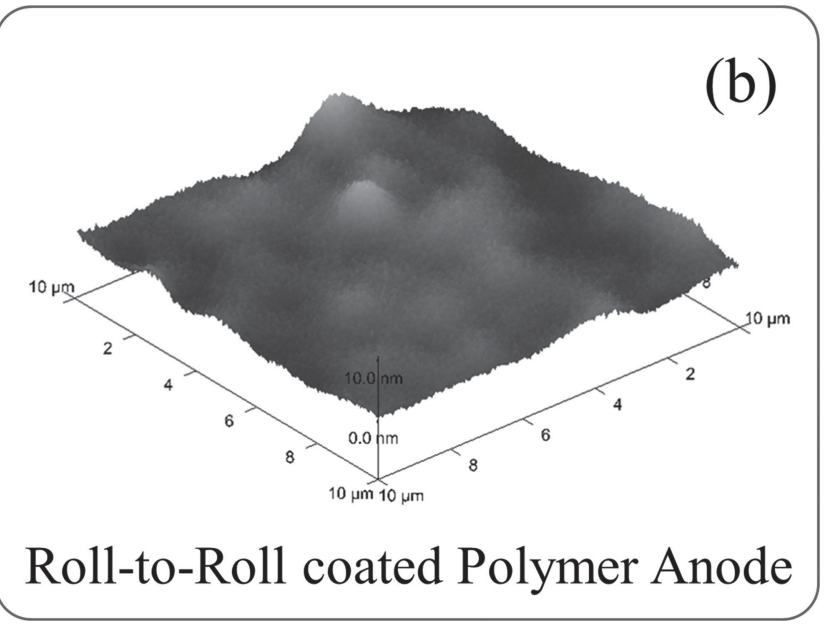

Figure 5. Comparison of atomic force microscopic images of conducting polymer anode and ITO anode acquired by AFM tapping mode. (a) The morphology of commercialized ITO-PET(15 $\Omega$ /square) film. (b) Conducting polymer (PH1000 modified with 5 wt $\%$ DMSO) anode. The two devices were measured under the same condition and the measured area was $(10 \mu \mathrm{m} \times 10 \mu \mathrm{m})$. 


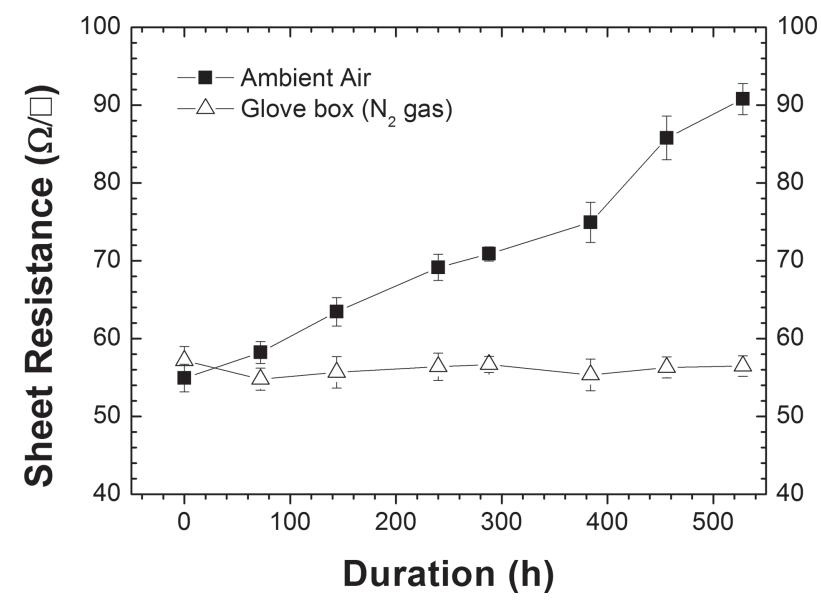

Figure 6. Durability of the conducting polymer anode maintained under the ambient air and inert gas $\left(\mathrm{N}_{2}\right.$ glove box: below $1 \mathrm{ppm} \mathrm{O}_{2}$ concentration) conditions. Each point represents 4 samples using mean and standard deviation with error bar. The black squares show the sheet resistance variation of the device under the ambient air, and the white triangles represent the sheet resistance variation of the device in the glove box.

the performance of transparent electrode and its effect on PLEDs performances.

Furthermore, this research is more focusing on the practical fabrication methods than higher efficiency device. For fair evaluation of the device performance in this paper, we selected SY as a light emitting polymer because it was not only commercialized but also commonly used as the evaluating material for comparing the device performances. However, because all layers including the electron injection layer of the present device were roll-to-roll coated under ambient air conditions, the device performance of the roll-to-roll coated device is hard to compare directly with other existing results.

In the conventional PLEDs, the ITO is commonly used as a transparent anode because it has high transparency and low sheet resistance at the same time. Furthermore, the ITO is used for high-efficiency PLEDs. However, the ITO has several problems, as mentioned above. In this study, we demonstrated that the performance of the polymer anode was superior to that of the ITO anode, through a comparison of the two. For a more accurate performance assessment of the ITO and polymer anodes, the two devices were subjected to the same process equally.

As a result, the turn-on voltages and the maximum luminance of the two devices were almost the same. In addition, the maximum luminous power efficiency of the polymeranode PLEDs was much higher than that of the ITO-anode PLEDs. Figure 7(a) shows the luminance and current densities of PLEDs following the input voltages. In case of the maximum luminance, the polymer-anode PLEDs reached $13090 \mathrm{~cd} / \mathrm{m}^{2}$ at $6.7 \mathrm{~V}$ and the ITO-anode PLEDs were $16370 \mathrm{~cd} / \mathrm{m}^{2}$ at $6.3 \mathrm{~V}$ with a same area of $4.4 \mathrm{~mm}^{2}$. The voltages of maximum luminance between the two devices were almost the same, implying that the thickness of each layer in the two devices was the same. The maximum luminance of the ITO-anode PLEDs was greater than that of the polymeranode PLEDs. However, the polymer-anode PLEDs showed excellent performance in a range of commercial operations, about 2 to $6 \mathrm{~V}$. Actually, the maximum luminance is of secondary importance because commercialized devices are not used under extreme conditions such as the maximum luminance. In addition, the leakage current of the polymeranode PLEDs was lower than that of the ITO-anode PLEDs due to improved surface roughness.

Figure 7(b) represents the maximum efficiency difference of the PLEDs fabricated with the polymer and ITO anodes. The luminous power efficiency was $4.13 \mathrm{~lm} / \mathrm{W}$ and $3.21 \mathrm{~lm} / \mathrm{W}$ for the polymer and ITO anodes, respectively, as shown in Figure 7(b). These results were conducted under the conditions listed in Table 3. Consequently, the PLEDs composed of a polymer anode exhibited $28 \%$ enhanced performance because the polymer anode presents a higher transparency than the ITO anode at a wavelength of $560 \mathrm{~nm}$, which are the peak position of electro luminance spectra of the SY. Furthermore, the extremely high roughness helped enhance the device efficiency, because it reduced the leakage current between the anode and the cathode.

The performance of the device fabricated by the roll-toroll cohesive coating method exhibited significant improvements as compared to the spin-coated device on a PET substrate, as shown in Figure 8. The current efficiency of the
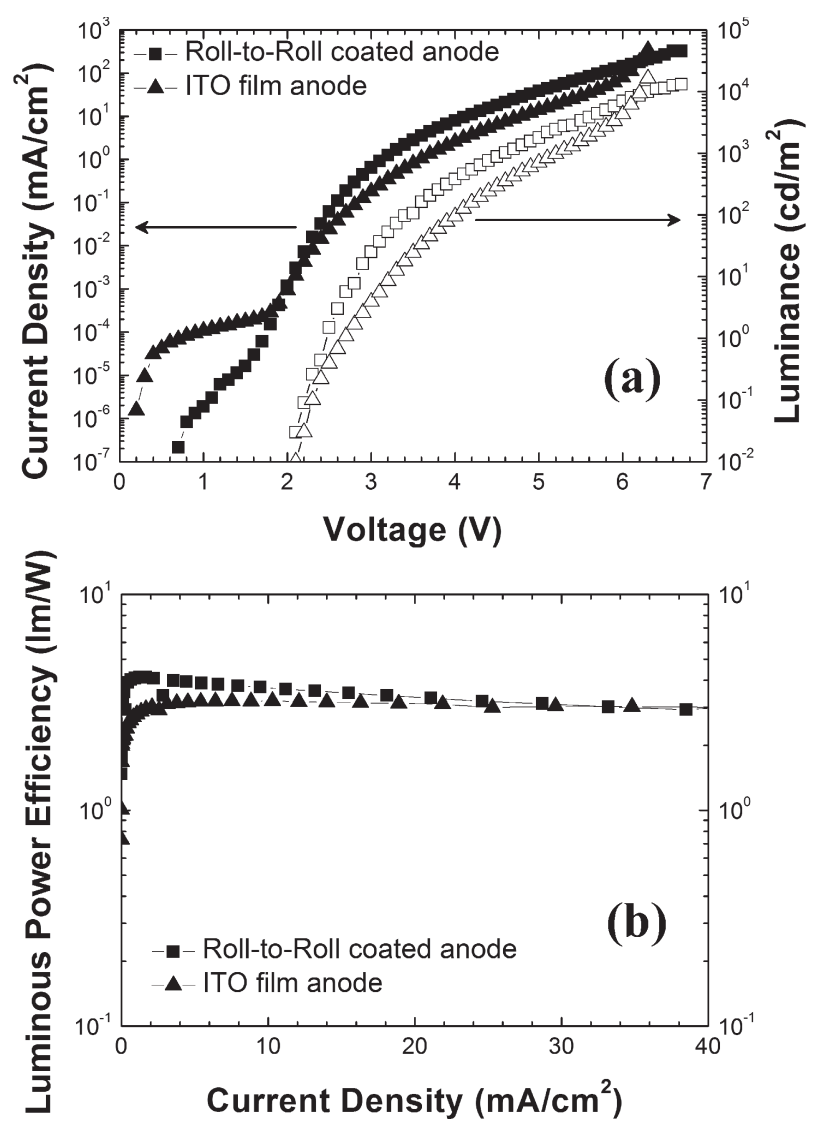

Figure 7. Comparison between the performances of ITO-anode PLEDs and conducting polymer-anode PLEDs. (a) Current density, voltage, and luminance (J-V-L) characteristics. The conducting polymer shows a lower current leakage before the turn-on voltage than does the ITO anode and matched maximum luminance. (b) Luminous power efficiency. The efficiency of the conducting polymer is better than that of ITO. 
Table 3. The roll-to-roll cohesive coating parameters and optimal conditions for proper thickness and high-efficiency PLEDs.

\begin{tabular}{lccccc}
\hline & PH1000 & Al4083 & SY & ZnO NPs & $\begin{array}{c}\text { Ionic } \\
\text { Solution }\end{array}$ \\
\hline $\begin{array}{l}\text { Roll speed } \\
{[\mathrm{mm} / \mathrm{s}]}\end{array}$ & 15 & 15 & 15 & 15 & 15 \\
$\begin{array}{l}\text { Spacer gap } \\
{[\mu \mathrm{m}]}\end{array}$ & 70 & 70 & 70 & 70 & 70 \\
Slit gap $[\mu \mathrm{m}]$ & 280 & 100 & 210 & 210 & 350 \\
\hline
\end{tabular}

optimized device exceeded $6.1 \mathrm{~cd} / \mathrm{A}$. This result is comparable to that of the ITO-glass $(10 \Omega / \square)$ device. The typical current efficiency of the ITO-glass is $6.3 \mathrm{~cd} / \mathrm{A}$, as shown in Figure S1, and the power efficiency of the optimized device was $5.1 \mathrm{~lm} / \mathrm{W}$ at a voltage of $4.3 \mathrm{~V}$. The current density of the roll-to-roll coated device below $2 \mathrm{~V}$ is lower than that of the ITO-glass. The surface of the polymer anode is smoother than that of the ITO-glass. The typical average surface roughness of the ITO-glass was $2.3 \mathrm{~nm}$, as shown in Figure S2.
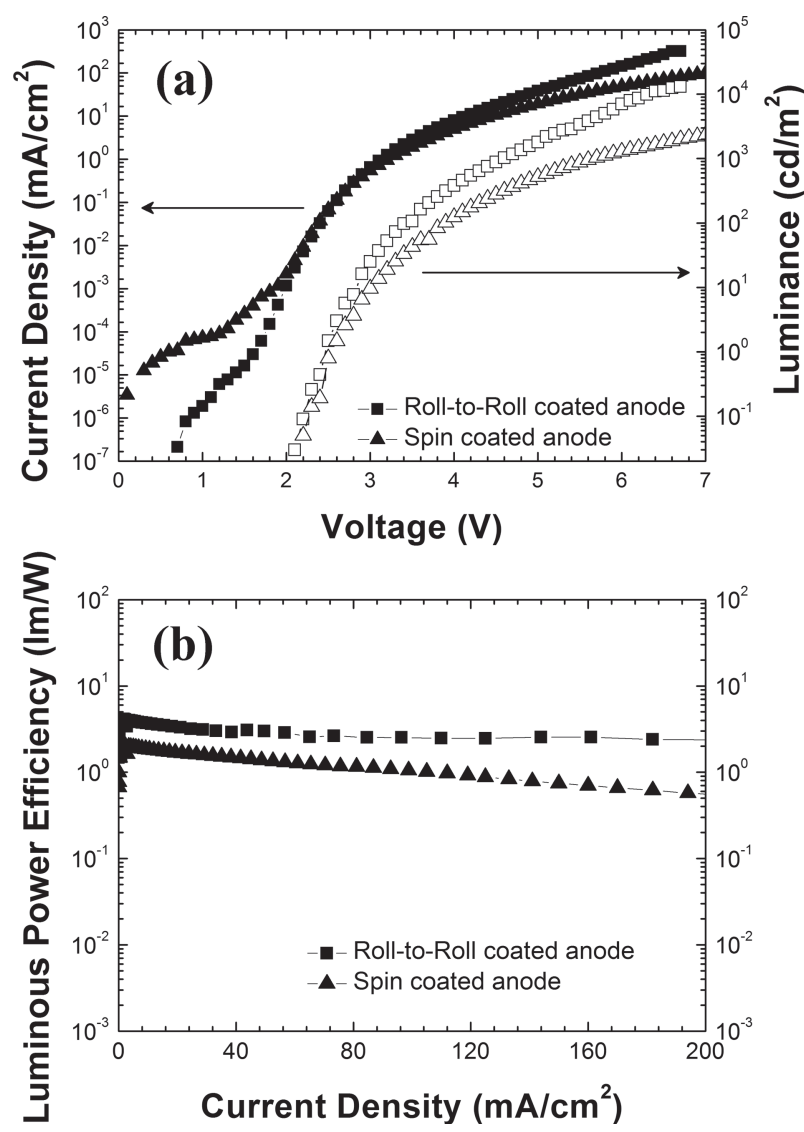

Figure 8. Comparison between the performances of the spin-coated polymer anode PLEDs and the roll-to-roll-coated polymer anode PLEDs on PET substrate. (a) Current density, voltage, and luminance (J-V-L) characteristics. The conventional spin-coating method shows poor performance as compared with the roll-to-roll cohesive coating method in all factors. (b) Luminous power efficiency.

\section{Conclusion}

We successfully demonstrated that high-efficiency polymer light-emitting diodes fabricated by a facile roll-to-roll coating method under ambient conditions. The PEDOT:PSS (PH1000, 5 wt\% DMSO) polymer anode, hole injection layer (AI4083), polymer light-emitting layer (Super Yellow), electron transport layer ( $\mathrm{ZnO}$ nanoparticle layer), and electron injection layer (ammonium ionic layer) were all fabricated by this roll-to-roll coating method. In particular, because all these materials have no alkali or alkaline earth metals, they can be fabricated in an air environment. This roll-toroll cohesive coating system employed a simple structured ink delivery system. The homogeneous ink supply from the blade and slit gap space significantly improved the coating quality. The novel roll-to-roll cohesive coating system in this work, utilizes only natural gravity and surface tension of the solution to flow out from the capillary to the surface of the substrate. Because this mechanism uses a minimally cohesive solution, the roll-to-roll cohesive coating can effectively reduce the flow rate and the wet-film thickness for the ultra-thin electron injection layer. In addition, the roll-to-roll cohesive coating enables us to make a thicker polymer anode film (more than $250 \mathrm{~nm}$ thick) at one time by modification of the surface energy and without wasting the solution. We observed that the sheet resistance of the roll-to-roll coated polymer anode had fewer variations during 50000 continuous bending cycles. During the bending cycles, the sheet resistance standard deviation of the polymer anode film was only $2.32 \Omega / \square$ (mean value of the sheet resistance: $66.06 \Omega / \square$ ).

The performance of roll-to-roll cohesive-coated PLEDs showed better performance as compared to the performance of spin-coated PLEDs. The luminous power efficiencies for the two devices were $4.13 \mathrm{~lm} / \mathrm{W}$ for the polymer anode-PET device and $3.21 \mathrm{~lm} / \mathrm{W}$ for the ITO-PET device. Consequentially, the PLEDs made by using the polymer anode exhibited $28 \%$ enhanced performance because the polymer anode represents not only a higher transparency than the ITO-PET at a wavelength of $560 \mathrm{~nm}$ but also significantly reduced roughness. The roll-to-roll cohesive coating method exhibited significant improvements over both the blade-only coating and spin-coating methods. Moreover, the optimized maximum current efficiency of the device was more than $6 \mathrm{~cd} / \mathrm{A}$. We expect that the roll-to-roll cohesive-coating method can be used as a practical approach in fabricating large-scale devices.

\section{Experimental Section}

Solution-processed PLEDs were fabricated on flexible PET substrates and the devices had the following structure: PEDOT:PSS (Clevios PH1000)-coated PET/PEDOT:PSS (Clevios PV P Al4083)/ Super Yellow (Merck PDY-132)/ZnO NPs and ionic solution/Al. The materials information of the PLEDs is as below. PH1000 is a polymer anode composed of PEDOT and PSS. The two materials are in a ratio of $1: 2.5$ (by weight) and the combined material has a specific conductivity of about $850 \mathrm{~S} / \mathrm{cm}$. Al4083 is type of PEDOT:PSS and serves as the hole injection material. The active polymer is phenyl-substituted poly( $p$-phenylene vinylene), known 
as "Super Yellow (SY)," dissolved in toluene at $0.5 \mathrm{wt} \%$. An electron transport layer is composed of zinc oxide ( $\mathrm{ZnO}$ ) nanoparticles (NPs) dissolved in 1-butanol at a concentration of $30 \mathrm{mg} / \mathrm{mL}$. The ZnO NPs were synthesized by chemical reaction described by Beek et al. ${ }^{[38]}$ and the typical particle size was approximately $5 \mathrm{~nm}$. The electron injection solution (ionic solution) consists of poly(ethylene oxide) and tetra- $n$-butyl ammonium tetrafluoroborate in acetonitrile. The concentrations of poly(ethylene oxide) and tetra- $\boldsymbol{n}$-butyl ammonium tetrafluoroborate in the ionic solution were 0.5 wt $\%$ and 0.2 wt $\%$, respectively.

The fabrication processes are as follows. Every layer was fabricated by the roll-coating process at a room temperature of $20^{\circ} \mathrm{C}$ under ambient air conditions. The spacer, which was a polyimide film about $70 \mu \mathrm{m}$ thick, was attached to both edges of the PET at every step. First, a polymer anode approximately $160 \mathrm{~nm}$ thick was deposited on the PET substrates and dried for $5 \mathrm{~min}$ at $120{ }^{\circ} \mathrm{C}$ in ambient air. The hole injection material, Al4083, was coated onto the PH1000-coated PET substrates and also dried at the same conditions. The final thickness of the Al4083 was approximately $40 \mathrm{~nm}$. Next, an emitting polymer (SY) with a thickness of approximately $75 \mathrm{~nm}$ was also roll-coated onto the Al4083 layer. The SY coated layer was annealed for $2 \mathrm{~h}$ at $75^{\circ} \mathrm{C}$ in a glove box. Finally, as the electron transport and injection materials, the ZnO NPs and the ionic solution were deposited on the SY layer. The entire thickness of the ZnO NP and ionic complex layer was about $30 \mathrm{~nm}$. The aluminum cathode $(120 \mathrm{~nm})$ was deposited by thermal evaporator under $2 \times 10^{-6}$ Torr. The detailed conditions of the roll-coating processes are represented in Table 3.

\section{Supporting Information}

Supporting Information is available from the Wiley Online Library or from the author.

\section{Acknowledgements}

This research was supported by Fostering Next-generation Researchers Program through the National Research Foundation of Korea (NRF) funded by the Ministry of Education, Science and Technology (D00016). The title of this article was changed after publication.

[1] Z. Chen, B. Cotterell, W. Wang, E. Guenther, S. Chua, Thin Solid Films 2001, 394, 201-205.

[2] S. R. Forrest, Nature 2004, 428, 911-918.

[3] S. T. Lee, Z. Q. Gao, L. S. Hung, Appl. Phys. Lett. 1999, 75, 1404-1406.

[4] D. Zhang, K. Ryu, X. Liu, E. Polikarpov, J. Ly, M. E. Tompson, C. Zhou, Nano Lett. 2006, 6, 1880-1886.

[5] J. Li, L. Hu, L. Wang, Y. Zhou, G. Gruner, T. J. Marks, Nano Lett. 2006, 6, 2472-2477.

[6] D. S. Hecht, L. Hu, G. Irvin, Adv. Mater. 2011, 23, 1482-1513.

[7] Z. Yu, Q. Zhang, L. Li, Q. Chen, X. Niu, J. Liu, Q. Pei, Adv. Mater. $2011,23,664-668$.
[8] L. Li, Z. Yu, W. Hu, C. H. Chang, Q. Chen, Q. Pei, Adv. Mater. 2011, 23, 5563-5567.

[9] Z. Yu, L. Li, Q. Zhang, W. Hu, Q. Pei, Adv. Mater. 2011, 23, 4453-4457.

[10] Y. M. Chang, L. Wang, W. F. Su, Org. Electron. 2008, 9, 968-973.

[11] Y. H. Ha, N. Nikolov, S. K. Pollack, J. Mastrangelo, B. D. Martin, R. Shashidhar, Adv. Funct. Mater. 2004, 14, 615-622.

[12] J. E. Yoo, K. S. Lee, A. Garcia, J. Tarver, E. D. Gomez, K. Baldwin, Y. Sun, H. Meng, T. Q. Nguyen, Y. L. Loo, Proc. Natl. Acad. Sci. USA 2010, 107, 5712-5717.

[13] J. Chung, B. Choi, H. H. Lee, Appl. Phys. Lett. 1999, 74, 3645-3647.

[14] Y. Yang, A. J. Heeger, Appl. Phys. Lett. 1994, 64, 1245-1247.

[15] J. Ouyang, C. W. Chu, F. C. Chen, Q. Xu, Y. Yang, Adv. Funct. Mater. 2005, 15, 203-208.

[16] P. A. Levermore, R. Jin, X. Wang, L. Chen, D. D. C. Bradley, J. C. de Mello, J. Mater. Chem. 2008, 18, 4414-4420.

[17] W. H. Kim, A. J. Makinen, N. Nikolov, R. Shashidhar, H. Kim, Z. H. Kafafi, Appl. Phys. Lett. 2002, 80, 3844-3846.

[18] C. J. Liang, H. Zou, Z. Q. He, C. X. Zhang, D. Li, Y. S. Wang, Chin. Phys. Lett. 2010, 27, 097801.

[19] P. Kopola, M. Tuomikoski, R. Suhonen, A. Maaninen, Thin Solid Films 2009, 517, 5757-5762.

[20] D.-H. Lee, J. S. Choi, H. Chae, C.-H. Chung, S. M. Cho, Displays 2008, 29, 436-439.

[21] J. Alstrup, M. Jørgensen, A. J. Medford, F. C. Krebs, ACS Appl. Mater. Interfaces 2010, 2, 2819-2827.

[22] Q. Xu, J. Ouyang, Y. Yang, Appl. Phys. Lett. 2003, 83, 4695-4697.

[23] J. Huang, G. Li, E. Wu, Q. Xu, Y. Yang, Adv. Mater. 2006, 18, 114-117.

[24] H. Wu, F. Huang, Y. Mo, W. Yang, D. Wang, J. Peng, Y. Cao, Adv. Mater. 2004, 16, 1826-1830.

[25] Y. Zhang, F. Huang, Y. Chi, A. K. Y. Jen, Adv. Mater. 2008, 20, $1565-1570$

[26] S. H. Oh, D. Vak, S. I. Na, T. W. Lee, D. Y. Kim, Adv. Mater. 2008, 20, 1624-1629.

[27] H. Youn, M. Yang, Appl. Phys. Lett. 2010, 97, 243302.

[28] B. H. Lee, S. H. Park, H. Back, K. Lee, Adv. Funct. Mater. 2011, 21, 487-493.

[29] S. R. Tseng, H. F. Meng, K. C. Lee, S. F. Horng, Appl. Phys. Lett. 2008, 93, 153308.

[30] C. Y. Chen, H. W. Chang, Y. F. Chang, B. J. Chang, Y. S. Lin, P. S. Jian, H. C. Yeh, H. T. Chien, E. C. Chen, Y. C. Chao, H. F. Meng, H. W. Zan, H. W. Lin, S. F. Horng, Y. J. Cheng, F. W. Yen, I. F. Lin, H. Y. Yang, K. J. Huang, J. Appl. Phys. 2011, 110, 094501.

[31] A. Sandstrom, H. F. Dam, F. C. Krebs, L. Edman, Nat. Commun. 2012, 3, 1002.

[32] J. Alstrup, M. Jørgensen, A. J. Medford, F. C. Krebs, ACS Appl. Mater. Interfaces. 2010, 2, 2819-2827.

[33] R. L. Bruce, F. Weilnboeck, T. Lin, R. J. Phaneuf, G. S. Oehrlein, B. K. Long, C. G. Wilson, J. J. Vegh, D. Nest, D. B. Graves, J. of Appl. Phys. 2010, 107, 084310.

[34] K. B. Kim, Y. H. Tak, Y. S. Han, K. H. Baik, M. H. Yoon, M. H. Lee, Jap. J. of Appl. Phys. 2003, 42, L438-L440.

[35] Z. Wang, S. Naka, H. Okada, Thin Solid Films. 2009, 518, 497-500.

[36] Y. Zhang, F. Huang, Y. Chi, A. K. Y. Jen, Adv. Mater. 2008, 20, 1565-1570.

[37] T. Ye, S. Shao, J. Chen, L. Wang, D. Ma, ACS Appl. Mater. Interfaces. 2011, 3, 410-416.

[38] W. J. E. Beek, M. M. Wienk, M. Kemerink, X. Yang, R. A. J. Janssen, J. Phys. Chem. B. 2005, 109, 9505.

Received: February 4, 2013

Revised: March 20, 2013

Published online: June 19, 2013 